\title{
Pancreas-visceral fat $C T$ value ratio and serrated pancreatic contour are strong predictors of postoperative pancreatic fistula after pancreaticojejunostomy
}

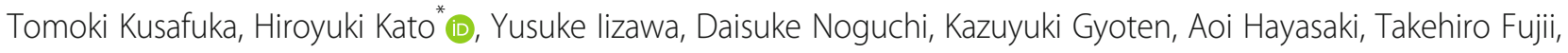
Yasuhiro Murata, Akihiro Tanemura, Naohisa Kuriyama, Yoshinori Azumi, Masashi Kishiwada, Shugo Mizuno, Masanobu Usui, Hiroyuki Sakurai and Shuji Isaji

\begin{abstract}
Background: Our aim is to elucidate the true preoperative risk factors for postoperative pancreatic fistula (POPF) after pancreaticoduodenectomy (PD), making it possible to select POPF high-risk patients preoperatively regardless of intraoperative pancreatic consistency judged by the surgeon's hand.

Methods: Among the 298 patients who underwent PD with pancreaticojejunostomy from 2007 to 2016, 262 patients had preoperative CT configurations that could be precisely evaluated. Risk factor analyses were conducted using various perioperative factors, including preoperative $C T$ findings, such as $C T$ values of the pancreas, pancreasvisceral fat $C T$ value ratio and pancreatic outer contour. Pancreatic outer contour was further divided into smooth(smooth interlobular) and serrated-type contours (feathery, irregular interlobular) by preoperative $C T$.

Results: In terms of the incidence of POPF, among the 262 patients, POPF grade B/C was found in 27 (10.3\%): grade B in 23 (8.8\%) and grade $C$ in 4 (1.5\%). According to multivariate analysis, a high pancreas-visceral fat $C T$ value ratio $(p=0.002)$, serrated-type contour $(p=0.02)$ and no history of chemoradiotherapy $(p=0.019)$ were identified as independent risk factors for POPF grade B/C. Even in patients with soft pancreas, the incidence of POPF grade B/C was $0 \%(0 / 57)$ in patients with a pancreas-visceral fat $C T$ value ratio of less than -0.4 and smooth-type contour, whereas the incidence was markedly high $(45.0 \%, 9 / 20)$ in patients with a pancreas-visceral fat $C T$ value ratio of -0.4 or greater and serrated-type contour, indicating that patients with soft pancreas should be categorized into POPF high-risk and low-risk groups according to preoperative $C T$ scan results.
\end{abstract}

Conclusions: The pancreas-visceral fat $C T$ value ratio and serrated-type pancreas are useful markers to preoperatively identify true POPF high-risk groups in patients undergoing PD, regardless of the pancreatic texture judged intraoperatively.

Keywords: BMl, Albumin, Pancreatic parenchymal $\subset$ value, Visceral fat $C T$ value

\footnotetext{
* Correspondence: katohiroyuki510719@gmail.com

Department of Hepatobiliary Pancreatic and Transplant Surgery, Mie University Graduate School of Medicine, 2-174 Edobashi, Tsu, Mie 514-8507, Japan
}

(c) The Author(s). 2020 Open Access This article is licensed under a Creative Commons Attribution 4.0 International License, which permits use, sharing, adaptation, distribution and reproduction in any medium or format, as long as you give appropriate credit to the original author(s) and the source, provide a link to the Creative Commons licence, and indicate if changes were made. The images or other third party material in this article are included in the article's Creative Commons licence, unless indicated otherwise in a credit line to the material. If material is not included in the article's Creative Commons licence and your intended use is not permitted by statutory regulation or exceeds the permitted use, you will need to obtain permission directly from the copyright holder. To view a copy of this licence, visit http://creativecommons.org/licenses/by/4.0/. The Creative Commons Public Domain Dedication waiver (http://creativecommons.org/publicdomain/zero/1.0/) applies to the data made available in this article, unless otherwise stated in a credit line to the data. 


\section{Background}

The probability of postoperative in-hospital mortality after pancreatoduodenectomy (PD) has decreased, especially in high-volume centres, with a mortality rate of less than $4 \%$ over recent decades [1,2]. A recent study using a national clinical database from Japan revealed that the 30-day and in-hospital mortality rates were 1.2 and $2.8 \%$, respectively [3]. Despite the fact that a low mortality rate has been observed, the incidence of clinically relevant postoperative pancreatic fistula (POPF: grade $\mathrm{B} / \mathrm{C}$ ), which most negatively affects patient outcome, has been recently reported to be $11-37 \%$ in patients with soft pancreas and $1-6 \%$ in patients with hard pancreas [4-9]. Regarding the risk factors for POPF, previous studies have reported various risk factors, such as age, sex, preoperative jaundice, operative time, intraoperative blood loss, type of pancreatic reconstruction, anastomotic technique, consistency of the pancreatic stump and pancreatic duct diameter [10-14], but there have been no reports focusing on preoperative computed tomography $(\mathrm{CT})$ configurations, especially the contour of the pancreas, for predicting POPF preoperatively.

The procedures of pancreatoenteral anastomosis have not been standardized, and each institution performs their own preferred procedure, such as pancreaticogastrostomy, pancreaticojejunostomy, external tube drainage, the lost stent method and invagination; this diversity of procedures makes it difficult to evaluate the frequency of POPF [15-17]. Our institution reported the method of 12 interrupted-stitched duct-to-mucosa pancreaticojejunostomy, named the "pair-watch suturing technique (PWST)", which allowed us to standardize the method of pancreaticojejunostomy [18-20]. However, even though the anastomotic technique has progressed, POPF still has yet to be thoroughly prevented after PD, and the incidence of POPF in patients with soft pancreas has been reported to be particularly high; thus, the prevention of POPF in patients with soft pancreas is still under discussion [21-23].

Recently, Sugimoto M et al. [21] reported that a thick parenchyma, a small main pancreatic duct (MPD), and fatty infiltration determined by postoperative histology were strongly associated with clinically relevant POPF after PD, especially in patients with soft pancreas, which was judged by intraoperative findings, and the study showed the negative impact of fat infiltration into the pancreatic parenchyma. Because pancreatic texture and consistency can be determined only by intraoperative findings or postoperative histological examinations, a high-risk group of POPF patients, especially those with soft pancreas, cannot be identified preoperatively. To solve this problem, Kuwahara $\mathrm{T}$ et al. [24] showed the usefulness of preoperative endoscopic ultrasonographyelastography (EUS-EG), which made it possible to objectively assess tissue elasticity preoperatively and predict the development of POPF after PD. However, EUSEG is still an uncommon procedure for the preoperative assessment of pancreatic consistency, and we consider it indispensable to select true soft pancreas and POPF high-risk patient groups preoperatively based on pancreatic configurations, such as the MPD diameter and parenchymal thickness, and on CT attenuation values of the pancreas, such as visceral fat and other ratios. All these parameters are easily measurable by plain $\mathrm{CT}$ images.

In terms of the contour of the pancreas in preoperative $\mathrm{CT}$, the precise cause of significant changes in the irregularity of the borders of the pancreas is unknown. When we analysed CT images to evaluate the precise morphology of the pancreas in order to investigate the type of pancreas that is likely to develop POPF, we noticed that 20 to $30 \%$ of patients with a mostly normal pancreas had irregular pancreatic borders, so we called this type of pancreas a serrated pancreas. According to previous research, a serrated pancreatic border is reported to be the result of ageing or acute weight loss after reversal of type 2 diabetes mellitus (DM) treated by a low-calorie diet [25]. This report also showed that the pancreas of patients with type $2 \mathrm{DM}$ obviously had more pancreatic marginal irregularities compared with the pancreas of healthy patients. In the field of diabetic internal medicine, pancreatic contour has been sometimes discussed, but there have been no studies evaluating the relationship between pancreatic outer contour and POPF after PD. Moreover, we hypothesized that a serrated pancreatic contour was associated with intralobular frailty, which results in parenchymal vulnerability during pancreaticojejunostomy. This vulnerability might result in difficulties associated with the anastomosis, with determining the risk of POPF regardless of the MPD size and pancreatic thickness, and with surgeons anastomosis skills.

In the present study, we evaluated 262 patients who underwent PD with PWST and analysed the pre- and intraoperative risk factors for POPF in these patients, focusing on the association between the incidence of POPF and the preoperative CT configuration of the pancreas as well as the CT attenuation values of the pancreatic parenchyma, visceral fat and these ratios. Our aim was to elucidate the true preoperative risk factors for POPF even in patients with soft pancreas, making it possible to select POPF high-risk patients preoperatively regardless of intraoperative pancreatic consistency judged by the surgeon's hand.

\section{Methods}

\section{Patients}

Among 319 patients who underwent PD from April 2007 to December 2016, PWST was performed in 298; there were 262 patients in the present study in whom the preoperative CT configuration could be precisely 
evaluated (Fig. 1). We retrospectively analysed the perioperative factors of POPF grade $\mathrm{B} / \mathrm{C}$, including various preoperative CT configurations. The study protocol was approved by the medical ethics committee of Mie University Hospital (No. 2857), and the study was performed in accordance with the ethical standards established in the 1964 Declaration of Helsinki.

\section{Surgical procedure}

For pancreaticojejunostomy, the first-layer anastomosis, which was a duct-to-mucosa anastomosis, was performed using PWST with 6-0 PDS II (Ethicon, Inc. Somerville, NJ, USA). This technique was conducted using 12 interrupted sutures oriented in a clock formation regardless of the MPD diameter [18-20]. This can be imagined as the faces of a pair of wristwatches, with the jejunal hole corresponding to the left-hand watch and the pancreatic duct hole to the right-hand one. The posterior wall of the pancreatic duct consists of the latter half of the clock cycle, from 6 to 12 o'clock, and the posterior wall of the jejunal hole consists of the first half of the clock cycle, from 12 to 6 o'clock. The secondlayer anastomosis was a pancreatic parenchymal-jejunal seromuscular anastomosis, which was performed via interrupted sutures with 4-0 Vicryl. In this study, PWST was carried out in all 262 patients. The surgical procedures included conventional PD in 28 patients, pyloruspreserving PD (PPPD) in 6 patients and subtotal stomach-preserving PD (SSPPD) in 228 patients. Laparoscopic SSPPD was performed in 12 patients, all of whom underwent the reconstruction procedures of pancreaticojejunostomy and hepaticojejunostomy under minilaparotomy. Reconstruction was carried out via a modified Child's method. A 5 Fr pancreatic stent tube was placed in patients with soft pancreas and/or a narrow MPD according to the surgeon's decision. A feeding jejunostomy tube was placed intraoperatively for early postoperative enteral nutrition. A single abdominal drain was inserted through the foramen of Winslow near the site of pancreaticojejunostomy. A drain was removed until postoperative day (POD) 5, as long as the drain discharge was clear and the drain amylase level was not three times higher than the upper limit of the serum amylase level $(132 \mathrm{U} / \mathrm{ml})$. A somatostatin analogue was not prophylactically used for preventing POPF.

\section{Assessment of POPF}

POPF was defined and graded according to the International Study Group on Pancreatic Fistula classification [26]. In all patients, the amylase activities of the abdominal drainage fluid were measured on postoperative day (POD) 3 to 7. For the diagnosis of POPF, any measurable volume of drainage fluid with an amylase level $>3$ times the upper limit of normal amylase $(132 \mathrm{U} / \mathrm{l})$ was considered the necessary threshold. POPF without any specific treatment despite the high drainage amylase level was categorized as biochemical leakage (BL). POPF was categorized as grade $\mathrm{B}$ when patients needed the following treatments: persistent drainage for more than 3 weeks, clinically relevant drain exchange, percutaneous or endoscopic drainage, and angiographic procedures. POPF was defined as grade $C$ when reoperation was performed or when organ failure developed due to POPF.

In this study, we focused on clinically relevant grade $\mathrm{B} / \mathrm{C}$ POPF. To identify the pre- and intraoperative risk factors for POPF, we compared various factors between patients with non-POPF or BL and those with POPF grade $\mathrm{B} / \mathrm{C}$.

\section{Measurements of CT attenuation values of the pancreatic parenchyma and visceral fat}

First, we conjecture that a lower CT attenuation value of the pancreatic parenchyma reflects fat deposition into the pancreas, a lower CT attenuation value of visceral fat

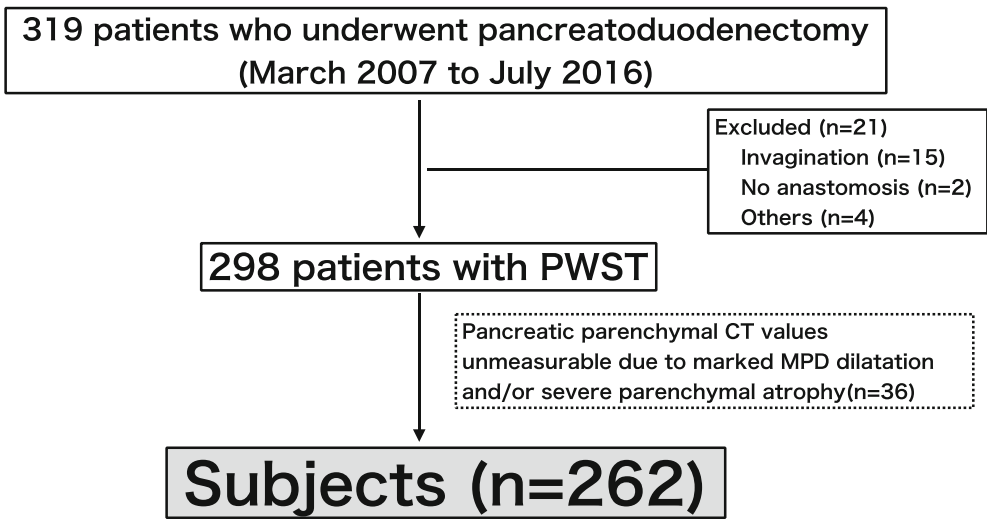

Fig. 1 Flow diagram of the subjects for the study. POPF: postoperative pancreatic fistula, PWST: pair-watch suturing technique, PDAC: pancreatic ductal adenocarcinoma, MPD: main pancreatic duct 
reflects adipose tissue hypertrophy, and these ratios might represent parenchymal quality. Thus, we measured these values and evaluated the association between these values and POPF grade B/C. Pancreatic parenchymal CT attenuation values in the future remnant pancreatic body/tail were measured at four different areas whose regions of interest (ROIs) were set by dragging the desired round area of 15 to $30 \mathrm{~mm}^{2}$ on a magnified CT image (Fig. 2a). To obtain accurate reproducibility, we concurrently used dynamic CT scans, including the arterial, portal and equilibrium phases, to exclude the areas of the pancreatic duct, splenic artery, splenic vein, portal vein and superior mesenteric artery. Visceral fat CT attenuation values were measured lateral to the stomach in four different areas whose ROIs were 15 to $30 \mathrm{~mm}^{2}$ (Fig. 2b). The mean CT value of the 4 different points of ROIs was employed for each CT scan. The pancreas-visceral fat $\mathrm{CT}$ value ratio was calculated as the mean pancreatic parenchymal CT value/mean visceral fat CT value. The MPD diameter was measured on CT at the planned resection level, and the pancreatic parenchymal thickness at the planned resection level was calculated by the following formula: the thickness of the pancreas (mm) - MPD diameter (mm).

\section{Configuration of the pancreatic outer contour determined by preoperative plain CT scan}

To determine the significant morphology of the pancreas influencing the development of POPF, all 262 pancreatic margins were traced, and we categorized the pancreatic contour into smooth- and serrated-type contours. According to the plain CT scans, the smooth type was defined as a pancreas with a smooth interlobular border, and the serrated type was defined as a pancreas with a feathery, irregular interlobular border and with a protrusion shape of more than $3 \mathrm{~mm}$, as shown in Fig. 3. Regardless of the pancreatic configuration, such as the thickness and presence or absence of MPD dilatation, a serrated-type pancreas was found in all categories of pancreatic configurations, but it was more frequently seen in the normal pancreas than in the atrophic pancreas and/or the pancreas with MPD dilatation.

\section{Risk factor analysis for POPF}

Uni- and multivariate analyses were conducted to evaluate the risk factors for POPF grade $\mathrm{B} / \mathrm{C}$ using pre- and intraoperative factors individually. The preoperative factors included age, sex, PDAC status, diabetes mellitus, Body mass index (BMI), history of chemoradiotherapy, MPD diameter on CT (mm), parenchymal thickness on CT (mm), pancreatic parenchymal CT value (Hounsfield units; $\mathrm{HU})$, visceral fat $\mathrm{CT}$ value (HU), pancreas-visceral fat $\mathrm{CT}$ value ratio and type of parenchymal contour. The intraoperative factors included the type of procedure, the use of laparoscopic surgery, combined portal vain (PV) resection, combined artery resection, combined distal pancreatectomy, pancreatic texture, MPD diameter judged intraoperatively $(\mathrm{mm})$, presence or absence of pancreatic stent tube, operation time, and intraoperative blood loss (ml).

\section{Statistical analysis}

All statistical analyses were performed using the statistical software package SPSS for Macintosh (version 24.0, IBM, Armonk, NY, USA). The results of the continuous variables were expressed as the median and range, and statistical significance was determined by the MannWhitney $U$ test. Discrete variables were evaluated by $x^{2}$ analysis or Fisher's exact test, as appropriate. The preand intraoperative risk factors associated with POPF

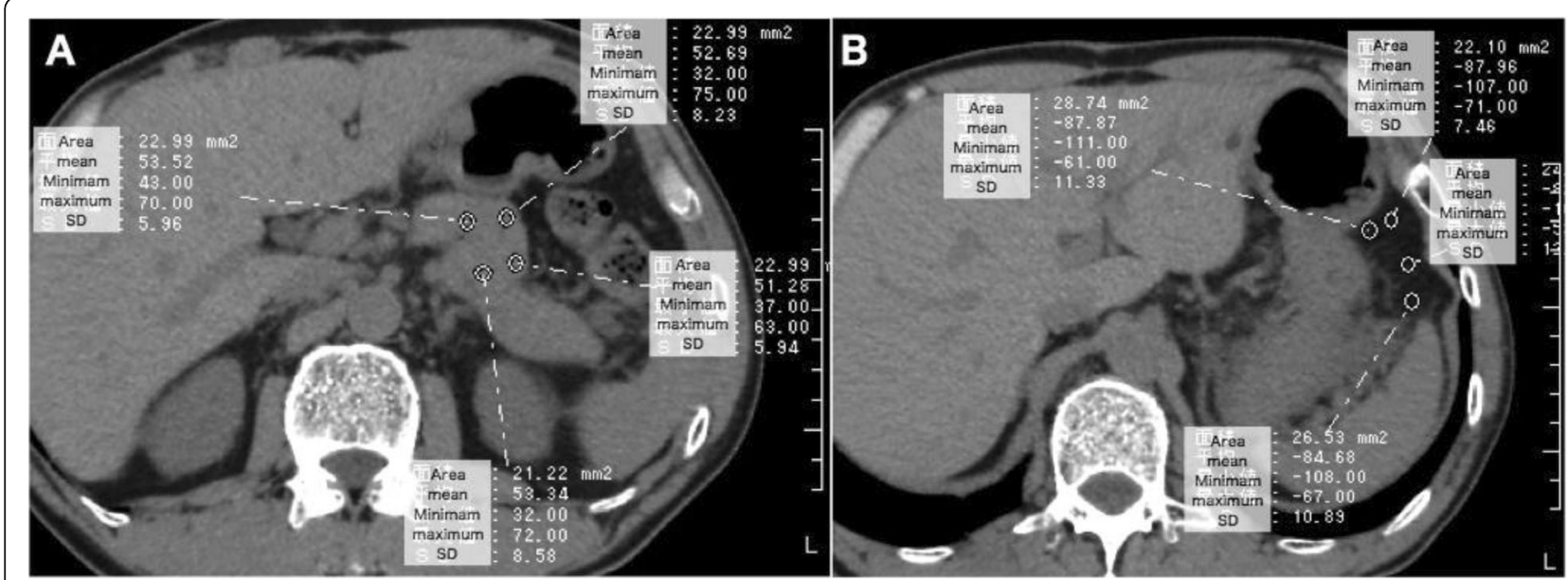

Fig. 2 Representative images for the measurements of CT values of the pancreatic parenchyma (a) and visceral fat (b). a Pancreatic CT values in the future remnant pancreatic body-tail are measured in the four different ROls area of 15 to $30 \mathrm{~mm} 2$ on a magnified $C T$ image. $\mathbf{b}$ Visceral fat CT values at lateral to the stomach are measured in the four different ROls area of 15 to $30 \mathrm{~mm} 2$. ROl: region of interest, SD: standard deviation 


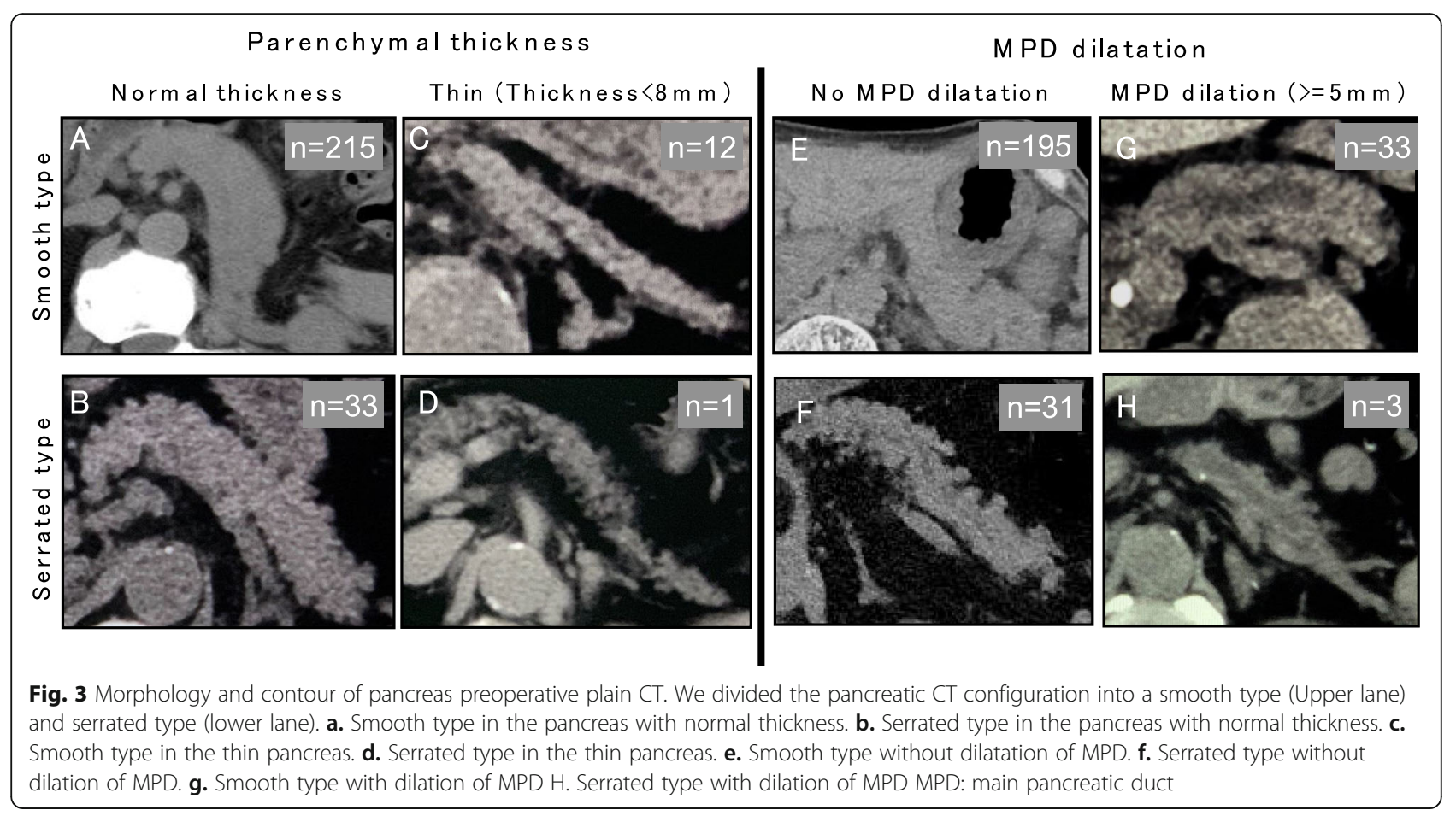

were analysed by uni- and multivariate analyses (multiregression analysis). Only variables whose $p$-values were less than 0.05 according to univariate analysis were included in the multivariate analysis. The results were considered significant when the $\mathrm{p}$-values were less than 0.05. The optimal cut-off value of the pancreas-visceral fat CT value ratio was determined using the diagnostic accuracy measurements and the receiver-operating characteristic (ROC) curves and was calculated on the basis of the maximum values of the Youden index, calculated by [sensitivity + specificity -1$]$.

\section{Results}

The characteristics of the subjects are shown in Table 1 . In these 262 patients, the median age (range) was 67.6 years old, and the male/female ratio was $158 / 104$. The primary diseases were pancreatic adenocarcinoma (PDAC) in 118 patients, intraductal papillary mucinous neoplasm (IPMN) in 52 patients, bile duct carcinoma in 53 patients and others in 39 patients. The results of the CT analysis were as follows: MPD diameter $(\mathrm{mm})=3.0$, pancreatic parenchymal thickness $(\mathrm{mm})=13.5$, pancreatic parenchymal CT value (Hounsfield Unit: $H U$ ) $=38.2$, visceral fat $\mathrm{CT}$ value $(\mathrm{HU})=-98.2$, and pancreasvisceral fat $\mathrm{CT}$ value ratio $=-0.39$, and the pancreatic texture judged intraoperatively was soft in 123 (46.9\%) and hard in 139 patients (53.1\%).
In terms of the type of pancreatic outer contour, the smooth type was observed in 228 (87\%) and the serrated type in 34 patients (13\%).

A pancreatic stent was placed in $140(53.4 \%)$ patients. The operation time $(\mathrm{min})$ and intraoperative blood loss (ml) were 526 and 863 , respectively. In terms of the incidence of POPF, among the 262 patients, clinically relevant $\mathrm{POPF}$, that is, $\mathrm{POPF}$ grade $\mathrm{B} / \mathrm{C}$, was found in 27 patients (10.3\%): grade B in $23(8.8 \%)$ and grade $C$ in 4 $(1.5 \%)$. For reference, BL was found in 20 patients (7.6\%). In terms of the treatment of POPF grade B, CTguided drainage was performed in 10 patients, reinitiation of antibiotics in 4, wound drainage in 3, drain exchange in 3 , angiography for bleeding in 2 and persistent drainage for more than 3 weeks in one. In the 4 patients with POPF grade $\mathrm{C}$, open laparotomy was performed in 3 patients, and mechanical ventilation for the treatment of acute lung injury was performed in one patient.

\section{Pre- and intraoperative risk factors for POPF}

As shown in Table 2, univariate analysis performed by comparing the preoperative risk factors between the POPF grade $\mathrm{B} / \mathrm{C}$ group and the non-POPF, $\mathrm{BL}$ group identified the following significant factors: male sex $(p=0.050)$, nonPDAC $(p=0.029)$, higher BMI $(p=0.002)$, absence of a history of chemoradiotherapy $(p=0.010)$, higher pancreatic parenchymal CT value $(p=0.028)$, lower visceral fat $\mathrm{CT}$ value $(\mathrm{HU})$, higher pancreas-visceral fat $\mathrm{CT}$ value ratio 
Table 1 Characteristics of the patients undergoing PD with PWST

\begin{tabular}{|c|c|}
\hline Patient's background & $n=262$ \\
\hline Age & $67.6(25-91)$ \\
\hline Gender: M / F & $158 / 104$ \\
\hline $\begin{array}{l}\text { Diagnosis: PDAC/IPMN/bile duct } \\
\text { cancer/others }\end{array}$ & $118 / 52 / 53 / 39$ \\
\hline $\begin{array}{l}\text { Preoperative diabetes mellitus } \\
\text { (yes/no) }\end{array}$ & $87 / 175$ \\
\hline BMI (kg/m2) & $22.2(14.1-40.0)$ \\
\hline Chemoradiotherapy (yes/no) & $98 / 164$ \\
\hline MPD diameter on CT (mm) & $3.0(1.0-12.6)$ \\
\hline $\begin{array}{l}\text { Pancreatic parenchymal thickness } \\
\text { on } C T^{*}(\mathrm{~mm})\end{array}$ & $13.5(4.5-27.0)$ \\
\hline Pancreatic parenchymal $C T$ value & $38.2(9.7-56.5)$ \\
\hline Visceral fat $C T$ value & $-98.2(-123.1 \sim-23.0)$ \\
\hline Pancreas-visceral fat $C T$ value ratio & $-0.39(-1.21 \sim-0.06)$ \\
\hline Procedure: PPPD/SSPPD/PD & $6 / 228 / 28$ \\
\hline $\begin{array}{l}\text { Surgeon's experience: }<20 \text { cases / } \\
20=<\text { cases }\end{array}$ & $180 / 82$ \\
\hline Laparoscopic surgery (yes/no) & $12 / 250$ \\
\hline Combined PV resection (yes/no) & $111 / 151$ \\
\hline $\begin{array}{l}\text { Pancreatic texture judged } \\
\text { intraoperatively (soft/hard) }\end{array}$ & $123 / 139$ \\
\hline Pancreatic stent (yes/no) & $140 / 122$ \\
\hline Operation time (min) & $526\left(286-1373^{a}\right)$ \\
\hline Intraoperative blood loss (ml) & $863\left(20-20,983^{b}\right)$ \\
\hline $\begin{array}{l}\text { Type of pancreatic contour } \\
\text { (smooth / serrated) }\end{array}$ & $228 / 34$ \\
\hline POPF: non/BL/B/C & $215 / 20 / 23 / 4$ \\
\hline
\end{tabular}

Parenchymal thickness $=$ the thickness of the pancreas $(\mathrm{mm})$ - MPD diameter $(\mathrm{mm})$, at the planned cut line

${ }^{a}$ This case underwent SSPPD, transverse colectomy and low anterior resection for triple cancer (duodenal papilla, transverse colon and rectum)

${ }^{\mathrm{b}}$ This case developed intraoperative massive bleeding due to the presence of intraabdominal abscess and severe adhesion to adjacent organs and vessels, but finally recovered

$P D A C$ pancreatic ductal adenocarcinoma, IPMN intraductal papillary mucinous neoplasm, $B M I$ body mass index, MPD main pancreatic duct, $P P P D$ pyloruspreserving pancreaticoduodenectomy, SSPPD subtotal stomach-preserving pancreaticoduodenectomy, $P D$ pancreaticoduodenectomy, $P V$ portal vein, POPF postoperative pancreatic fistula, $B L$ biochemical leak

$(p=0.00025)$, and serrated-type contour $(p<0.001)$. According to multivariate analysis, a higher pancreas-visceral fat CT value ratio $(p=0.002)$ and frequency of serratedtype contour $(p=0.020)$ and the lack of a chemoradiotherapy history $(p=0.019)$ were calculated as independent risk factors for POPF.

As shown in Table 3, the absence of combined PV resection $(\mathrm{p}=0.002)$ and soft pancreas $(p=0.001)$ were selected as significant intraoperative risk factors for POPF according to univariate analysis, and only soft pancreas $(p=0.050)$ was calculated as an independent risk factor for POPF.

\section{Risk categorization of POPF according to the pancreas-} visceral fat $\mathrm{CT}$ value ratio and pancreatic outer contour First, to clarify the clinical relevance of the pancreasvisceral fat $C T$ value ratio for predicting POPF after PD, the optimal cut-off point was determined using a receiver operating characteristic curve (ROC). As shown in Fig. 4a, the cut-off point of the pancreas-visceral fat CT value ratio was -0.40 (AUC: 0.711 ). Moreover, the incidence of POPF grade B/C was $18.2 \%(26 / 143)$ in patients with a value of -0.40 or greater, which was significantly higher than the incidence of $0.8 \%(1 / 119)$ in patients with a pancreas-visceral fat CT value ratio of less than $0.40(P<0.001)$, as shown in Fig. $4 b$. Figure $4 c$ shows the $2 \times 2$ contingency table analysis for predicting POPF patients divided based on the pancreas-visceral fat CT value ratio and pancreatic outer contour. The analysis revealed that the incidence of POPF grade $\mathrm{B} / \mathrm{C}$ was markedly high $(36.0 \%, 9 / 25)$ in patients with a pancreasvisceral fat CT value ratio of -0.4 or greater and serrated-type contour, whereas it was $0 \%(0 / 110)$ in patients with a pancreas-visceral fat CT value ratio of less than -0.4 and smooth-type contour. Therefore, the patients in whom PD was proposed could be categorized into the POPF high-risk group (pancreas-visceral fat CT value ratio $>=-0.40$ and serrated type) or low-risk group (pancreas-visceral fat CT value ratio $<-0.40$ and smooth type) according to these factors regardless of intraoperative pancreatic consistency.

\section{Association between pancreatic configuration and pancreatic texture judged intraoperatively}

The incidence of serrated-type contour was significantly higher in patients with soft pancreas than in patients with hard pancreas $(22 \%, 27 / 123$ vs. $5 \%, 7 / 139 . p<$ $0.001)$. On the other hand, the value of the pancreasvisceral fat $\mathrm{CT}$ value ratio tended to be lower in patients with soft pancreas than in patients with hard pancreas (median: -0.40 vs. $-0.38, p=0.066$ ).

Next, we focused on the association between the incidence of POPF and our predictors in only soft pancreatic patients because the intraoperative judgement of soft pancreas was the only significant factor predicting POPF. In the same manner as Fig. $4 \mathrm{c}$, the $2 \times 2$ contingency table analysis for predicting POPF patients divided based on the pancreas-visceral fat $\mathrm{CT}$ value ratio and pancreatic outer contour was conducted only for soft pancreatic texture. The analysis revealed that the incidence of POPF grade B/C was markedly high (45.0\%, 9/ 20) in patients with a pancreas-visceral fat $C T$ value ratio of -0.4 or greater and serrated-type contour, whereas the incidence was $0 \%(0 / 57)$ in patients with a 
Table 2 Univariate and multivariate analysis for evaluating preoperative risk factors associated with POPF

\begin{tabular}{|c|c|c|c|c|c|}
\hline Variables & $\begin{array}{l}\text { non-POPF, BL }(\boldsymbol{n}= \\
235)\end{array}$ & $\begin{array}{l}\text { POPF Grade BC } \\
(\boldsymbol{n}=27)\end{array}$ & $\begin{array}{l}\boldsymbol{P} \text {-value } \\
\text { (Univariate) }\end{array}$ & Odd's ratio $(95 \% \mathrm{Cl})$ & $\begin{array}{l}\boldsymbol{P} \text {-value } \\
\text { (Multivariate) }\end{array}$ \\
\hline Age & $67.0(25-89)$ & $67.0(25-89)$ & 0.094 & & \\
\hline Gender: M / F & $137 / 98$ & $21 / 6$ & 0.050 & - & - \\
\hline Diagnosis: (PDAC/non-PDAC) & $113 / 122$ & $7 / 20$ & 0.029 & - & - \\
\hline Preoperative diabetes mellitus (yes/no) & $81 / 154$ & $6 / 21$ & 0.201 & & \\
\hline BMI (kg/m2) & $21.1(14.9-40.0)$ & $23.7(14.1-31.0)$ & 0.002 & - & - \\
\hline Chemoradiotherapy (yes/no) & $94 / 141$ & $4 / 23$ & 0.010 & $0.25(0.08-0.80)$ & 0.019 \\
\hline MPD diameter on CT (mm) & $3.0(1.0-13.6)$ & $2.5(1.0-6.0)$ & 0.160 & & \\
\hline $\begin{array}{l}\text { Pancreatic parenchymal thickness on CT } \\
*(\mathrm{~mm})\end{array}$ & $13.5(4.5-27.0)$ & $14.0(25.0-25.0)$ & 0.348 & & \\
\hline Pancreatic parenchymal CT value (HU) & $38.5(10.2-56.5)$ & $35.8(9.7-54.8)$ & 0.028 & - & - \\
\hline Visceral fat $\mathrm{CT}$ value $(\mathrm{HU})$ & $\begin{array}{l}-97.5(-123.1-- \\
100.8)\end{array}$ & $\begin{array}{l}-100.8(-120.7- \\
-23.0)\end{array}$ & 0.025 & - & - \\
\hline pancreas-visceral fat $\mathrm{CT}$ value ratio & $\begin{array}{l}-0.40(-1.21- \\
-0.117)\end{array}$ & $-0.35(-0.42-0.09)$ & 0.00025 & $\begin{array}{l}2891.5(17.6-473 \\
225.1)\end{array}$ & 0.002 \\
\hline $\begin{array}{l}\text { Type of parenchymal contour (Serrated/ } \\
\text { smooth) }\end{array}$ & $24 / 211$ & $10 / 17$ & $<0.001$ & $3.11(1.20-8.06)$ & 0.020 \\
\hline
\end{tabular}

Parenchymal thickness = the thickness of the pancreas $(\mathrm{mm})$ - MPD diameter $(\mathrm{mm})$, at the planned cut line

$\mathrm{Cl}$ confidence interval, PDAC pancreatic ductal adenocarcinoma, IPMN intraductal papillary mucinous neoplasm, $B M I$ body mass index, $M P D$ main pancreatic duct, $P P P D$ pylorus-preserving pancreaticoduodenectomy, SSPPD subtotal stomach-preserving pancreaticoduodenectomy, $P O P F$ postoperative pancreatic fistula, $B L$ biochemical leak, Statistical analysis: Mann- Whitney $U$ test for contentious variables. $X^{2}$ analysis for discrete variables

pancreas-visceral fat CT value ratio of less than -0.4 and smooth-type contour (Table 4), proving that even patients with soft pancreas should be categorized into POPF high- (Table 4, red), intermediate- (Table 4, yellow) and low- (Table 4, green) risk groups according to the preoperative CT scans.
Histological evaluation of the pancreatic stump to estimate the percentage of parenchymal and interlobular (PI) area

To confirm whether our risk categorization based preoperative CT configurations reflects the quality of the pancreas, loupe images of the pancreatic stump with

Table 3 Univariate and multivariate analysis for evaluating intraoperative risk factors associated with POPF

\begin{tabular}{|c|c|c|c|c|c|}
\hline Variables & $\begin{array}{l}\text { non-POPF, BL }(n= \\
235)\end{array}$ & $\begin{array}{l}\text { POPF Grade BC } \\
(n=27)\end{array}$ & $\begin{array}{l}\text { P-value } \\
\text { (Univariate) }\end{array}$ & $\begin{array}{l}\text { Odd's ratio }(95 \% \\
\text { Cl) }\end{array}$ & $\begin{array}{l}\text { P-value } \\
\text { (Multivariate) }\end{array}$ \\
\hline Procedure: PPPD/SSPPD/PD & $6 / 203 / 26$ & $0 / 25 / 2$ & 0.577 & & \\
\hline Laparoscopic surgery (yes/no) & $9 / 226$ & $3 / 24$ & 0.087 & & \\
\hline Combined PV resection (yes/no) & $107 / 128$ & $4 / 23$ & 0.002 & $2.72(0.80-9.31)$ & 0.110 \\
\hline Combined artery resection (yes/no) & $9 / 226$ & $3 / 24$ & 0.087 & & \\
\hline Combined distal pancreatectomy (yes/no) & $3 / 232$ & $1 / 26$ & 0.330 & & \\
\hline $\begin{array}{l}\text { Pancreatic texture judged intraoperatively } \\
\text { (soft/hard) }\end{array}$ & $102 / 133$ & $21 / 6$ & 0.001 & $2.89(1.00-8.35)$ & 0.050 \\
\hline $\begin{array}{l}\text { Diameter of main pancreatic duct judged } \\
\text { intraoperatively }\end{array}$ & $4(1-15)$ & $3(2-8)$ & 0.054 & & \\
\hline Pancreatic stent (yes/no) & $123 / 112$ & $17 / 10$ & 0.654 & & \\
\hline Operation time (min) & $498.5\left(286-1373^{a}\right)$ & $496.0(333-670)$ & 0.591 & & \\
\hline Intraoperative blood loss (ml) & $713.0\left(20-20983^{b}\right)$ & $692.0(210-5522)$ & 0.234 & & \\
\hline
\end{tabular}

${ }^{a}$ This case underwent SSPPD, transverse colectomy and low anterior resection for triple cancer (duodenal papilla, transverse colon and rectum)

${ }^{\mathrm{b}}$ This case developed intraoperative massive bleeding due to the presence of intraabdominal abscess and severe adhesion to adjacent organs and vessels, but finally recovered

$\mathrm{Cl}$ confidence interval, PPPD pylorus-preserving pancreaticoduodenectomy, SSPPD subtotal stomach-preserving pancreaticoduodenectomy, $P D$ pancreaticoduodenectomy, PV portal vein, POPF postoperative pancreatic fistula, BL biochemical leak, Statistical analysis: Mann- Whitney U test for contentious variables. $X^{2}$ analysis for discrete variables 


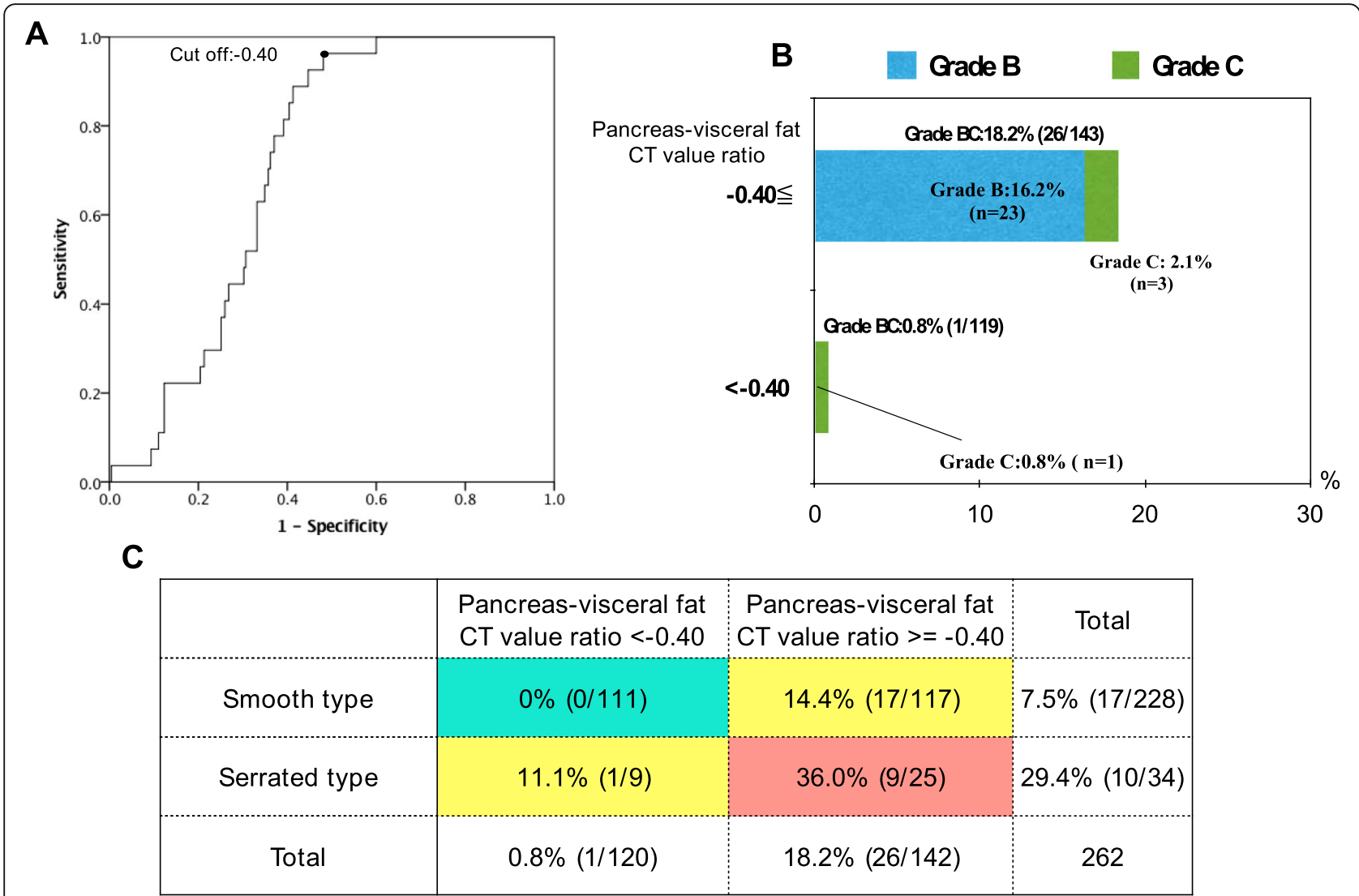

Fig. 4 Prediction of POPF in total 262 patients. a. Receiver operating characteristic (ROC) curve. Cut-off point of pancreas-visceral fat CT value ratio is -0.40 (AUC:0711). b. The incidence of POPF according to the pancreas-visceral fat CT value ratio. c. The $2 \times 2$ Contingency table analysis for the incidences of POPF according to pancreas-visceral fat $C T$ value ratio and pancreatic outer contour POPF: postoperative pancreatic fistula

haematoxylin and eosin staining were analysed in soft pancreatic patients by using their binary images with ImageJ software [27] in an attempt to estimate the percentage of the PI area and the degree of fat infiltration. In soft pancreatic patients with a high risk of POPF $(n=$ $20)$, the percentage of the PI area was significantly lower than that in patients with a low risk of POPF $(n=57)$ (68.2 vs $81.5 \%, p=0.00002$ ) (Fig. 5).

\section{Discussion}

In the present study, using a homogeneous cohort of patients who underwent pancreaticojejunostomy with PWST, we newly revealed that the pancreas-visceral fat CT value ratio, serrated-type pancreatic contour, and a history of chemoradiotherapy were strong preoperative predictors of POPF after PD. Furthermore, soft pancreatic texture was selected as the only intraoperative risk factor for POPF, and this result has been widely accepted among pancreatic surgeons until now. Among these risk factors, we considered that the pancreasvisceral fat $\mathrm{CT}$ value ratio and serrated-type pancreatic contour were closely associated with the degradation of pancreatic parenchymal quality characterized by parenchymal fat deposition, which was evidenced by histological evaluation of the parenchymal stump.

Since the effective management of POPF has proven to be a difficult challenge despite recent improvements in postoperative patient care, the early identification of POPF high-risk groups has made a paradigm shift among pancreatic surgeons from a reactive and passive approach that begins to treat POPF when it becomes apparent to a proactive approach that depends on early anticipation and timely prevention through prophylactic measures. However, this approach is predicated on the assumption that POPF high-risk groups can actually be predicted. To predict POPF more precisely, a clinical scoring system by Callery MP et al. [28] was considered to be very useful because the incidence of clinically relevant POPF reached more than $67 \%$ in patients with scores greater than seven, which consisted of gland texture, pathology (pancreatic adenocarcinoma or pancreatitis or others), pancreatic duct diameter and the amount of intraoperative blood loss. However, this score cannot be assessed preoperatively because the scoring systems include several intra- and postoperative variables, such as gland texture, pathological diagnosis and 
Table 4 The $2 \times 2$ Contingency table analysis for the incidences of POPF according to pancreas-visceral fat CT value ratio and pancreatic outer contour in the 123 patients with soft pancreatic texture

\begin{tabular}{|l|l|l|l|}
\hline & Soft and pancreas-visceral fat CT & Soft and pancreas-visceral fat CT & Total \\
& value ratio____- 0.40 & value ratio_-_=__- 0.40 & \\
\hline Soft and & $0 \%(0 / 57)$ low risk & $28.0 \%(11 / 39)$ intermediate risk & $11.4 \%$ \\
smooth type & $15.6 \%(1 / 7)$ intermediate risk & $45 \%(9 / 20)$ high risk & $(11 / 96)$ \\
\hline Soft and & & & $37.0 \%(10 / 27)$ \\
\hline serrated type & $1.5 \%(1 / 64)$ & $33.9 \%(20 / 59)$ & 123 \\
\hline
\end{tabular}

A

POPF low risk in soft pancreas $(n=57)$

( $\mathrm{Sm}$ ooth and pancreas-visceral fat $\mathrm{CT}$ value ratio $<-0.40$ )

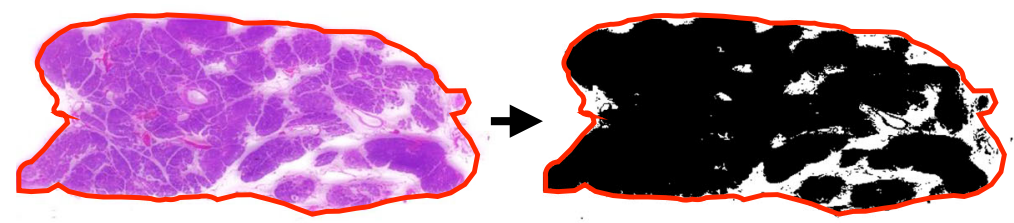

POPF high risk in soft pancreas $(n=20)$

(Serrated and Pancreas - visceral fat $C T$ value ratio $>=-0.40$ )
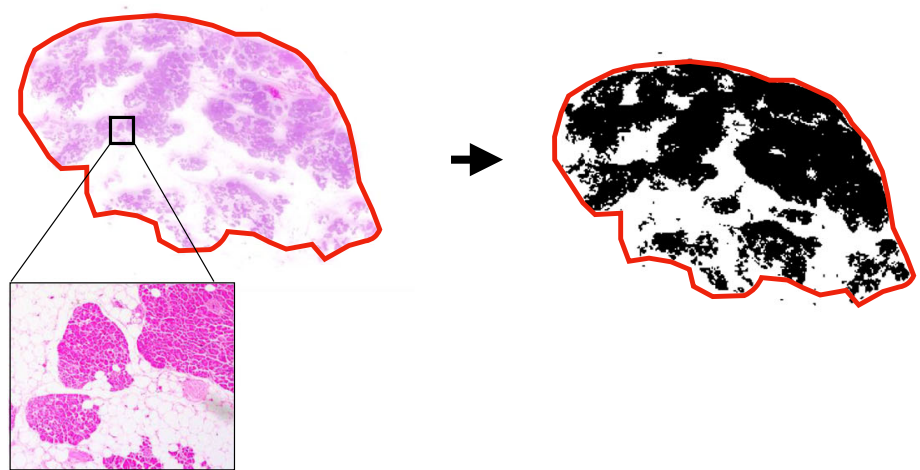

B

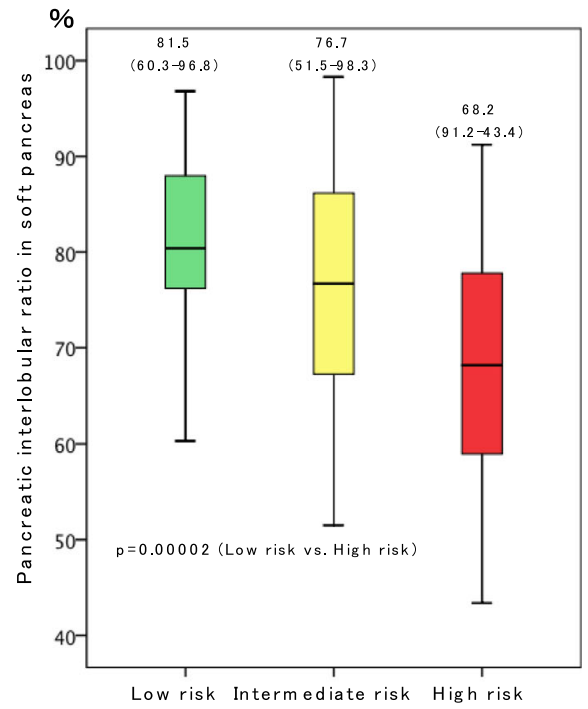

Fig. 5 Histological evaluation of the pancreatic stump to estimate the percentage of a parenchymal and interlobular (PI) area using ImageJ software. a. Loupe images of the pancreatic stump with hematoxylin and eosin staining and their binary images by ImageJ software. After the outer circumference of the entire cut surface (red line) is manually outlined, the entire cut surface area is measured by using ImageJ software. The black area is regarded as the PI area. The white area is regarded as the area including fatty tissue. Magnified pictures showed representative images according to the POPF low or high-risk groups in the soft pancreas. In a typical case with POPF low risk (upper picture of A), the percentage of Pl area/entire surface area was 80.0\% (252.1 / 315.8 × 100). On the other hand, in a typical case with POPF high risk (lower picture of A), the percentage of PI area/entire surface area was 52.6\% (132.6 / $252.1 \times 100)$. b. Box plot graph for the comparison of the percentage of PI area. It is significantly higher than in the POPF low-risk group than that of the high-risk group $(p=0.00002)$ 
blood loss. In particular, as pancreatic gland texture is judged by the surgeon's hand intraoperatively, this approach is a very subjective method; therefore, preoperative indicators for predicting POPF should be identified as an alternative to conducting the proactive approach. When we treat high-risk groups of patients, we should pay much more attention to the occurrence of POPF from the preoperative setting so that preoperative, careful informed consent can be provided to patients and their families. Postoperatively, we should carefully check the amylase levels of the drainage discharge and the appearance of fluid. If inflammatory reactions are unexpectedly escalated or continue in high-risk groups, an early follow-up CT scan should be conducted to detect the presence or absence of pancreatic fistula.

Among the various preoperative risk factors, the pancreas-visceral fat CT value ratio was selected as the most independent factor for predicting POPF rather than the CT value of the pancreatic parenchyma itself. We considered that the pancreas-visceral fat $\mathrm{CT}$ value ratio represents the quality of the pancreatic parenchyma. Kitajima $\mathrm{Y}$ et al. [29] measured intramuscular adipose tissue content (IMAC) using CT, and this measure has recently attracted much attention for evaluating the quality of skeletal muscle because several studies have revealed that increased IMAC is positively linked to worse survival after resection of PDAC [30] and to an increased complication rate after hepatectomy for hepatocellular carcinoma [31]. Nevertheless, there have been no reports regarding pancreatic parenchymal quality. In this study, we hypothesized that a lower quality of the pancreatic parenchyma might result in vulnerability of pancreaticojejunal anastomosis due to severe fat infiltration. Since IMAC is calculated by the ratio of the multifidus muscle to the subcutaneous fat $\mathrm{CT}$ attenuation value, we analysed whether the pancreas-visceral fat $\mathrm{CT}$ value ratio represented the quality of the pancreas by comparing the incidence of POPF, and this is the first report regarding the estimation of pancreatic parenchymal quality using plain CT images.

A history of chemoradiotherapy reduced the incidence of POPF in the present study. In general, preoperative chemoradiotherapy is considered to reduce POPF after pancreatectomy because radiation induces intralobular fibrosis and exacerbates its exocrine function [32, 33]. Moreover, in our institution, most candidates who undergo preoperative chemoradiotherapy are patients with advanced PDAC, in whom exocrine function is generally ruined due to MPD obstruction. As a result, preoperative chemotherapy significantly reduced POPF after PD in our cohort.

Serrated-type pancreatic contour determined by preoperative plain CT was also selected as another independent risk factor for POPF. In fact, the incidence of POPF was significantly higher in patients with a serrated-type pancreas $(29.4 \%, 10 / 34)$ than in patients with a smooth pancreas $(7.4 \%, 17 / 228)$. Indeed, serratedtype contour was mostly seen in patients with soft pancreas, whereas this type of contour was barely found in patients with a hard pancreatic texture. The precise aetiology of significant changes in the irregularity of the borders of the pancreas is unknown, but serrated-type pancreatic contour has been reported to be the result of ageing or acute weight loss after the reversal of type 2 DM treated by a low-calorie diet [25]. When we focused on basic research regarding pancreatic exocrine architectures, ghrelin, a hunger-stimulating hormone produced by the fundus of the stomach, increased exocrine pancreatic fractal dimensions and textural entropy and decreased the lacunarity of the acinar cell architecture in rats, regardless of age [34]. In the clinical setting, Sasaki $\mathrm{K}$ et al. [35] reported that the individual ghrelin ratio (POD1/prior to operation) was significantly lower in patients who developed complications, especially POPF and intraabdominal abscess, than in those who did not. The lack of ghrelin exertion and weight loss in patients

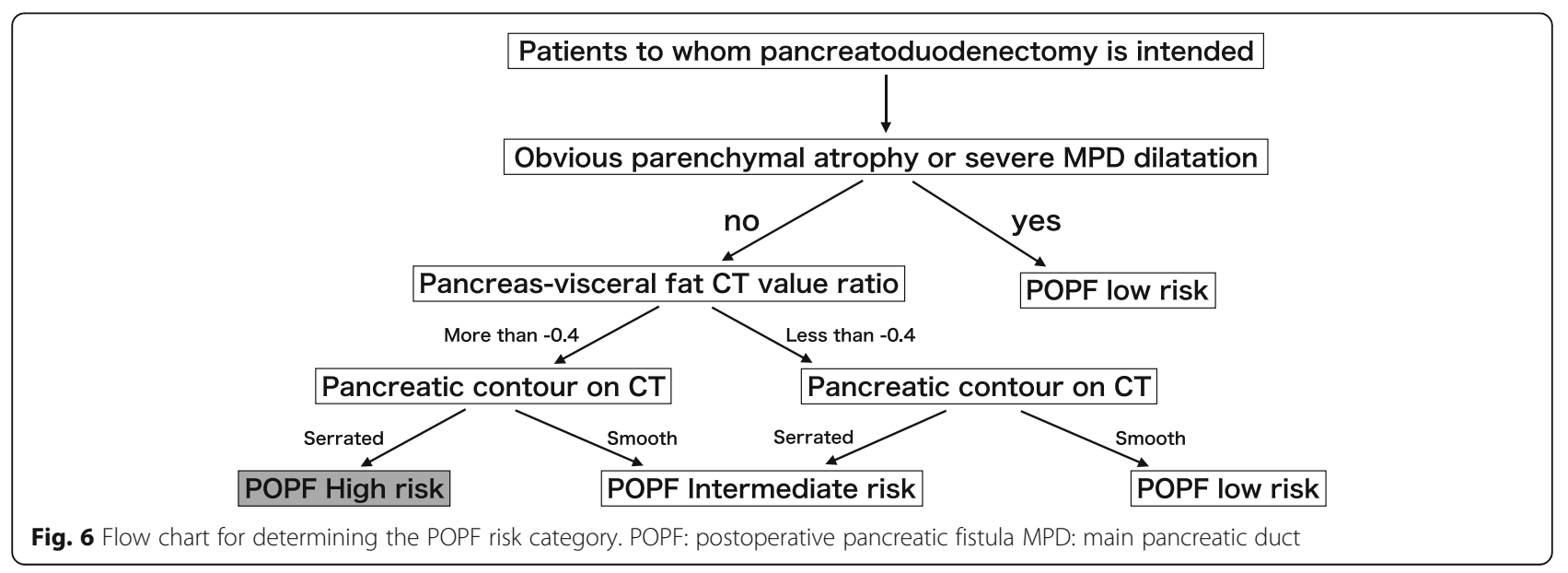


with type 2 DM might be related to the formation of a serrated-type pancreas and its parenchymal frailty, which significantly accelerate the incidence of POPF. In our present study, however, the incidence of a serrated-type pancreas was comparable between diabetic and nondiabetic patients, and the level of ghrelin in the blood was not measured. Therefore, further research is needed to elucidate the cause of serrated-type pancreatic contour.

Soft pancreas is generally characterized by a narrow pancreatic duct and vulnerable parenchymal texture, resulting in a high risk of POPF after PD. However, as shown in our $2 \times 2$ contingency table analysis (Table 4 ), the incidence of POPF grade $\mathrm{B} / \mathrm{C}$ was $0 \%(0 / 57)$ in patients with a pancreas-visceral fat $\mathrm{CT}$ value ratio of less than -0.4 and smooth-type contour, even though these patients were categorized as patients with soft pancreas intraoperatively. The high-risk group showed an obviously high incidence of POPF (9/20). Moreover, these pancreases were characterized by obvious fat infiltration pathologically, as shown in Fig. 5. Indeed, fatty pancreas is considered to be a high-risk factor for POPF, and Mathur A et al. [36] demonstrated that patients with increased fat and decreased fibrosis had a higher risk of POPF after PD. Gaujoux S et al. [37] also showed that an increased body mass index, fatty pancreas, and the absence of fibrosis were associated with a risk of POPF after PD. In these reports, the amount of fat deposition and degree of fibrosis were examined by histological findings, and therefore, these factors could not be assessed preoperatively. To assess the amount of pancreatic fat and its influence on POPF preoperatively, Lee SE et al. [38] suggested the usefulness of relative signal intensity decreases in preoperative dual-gradient-echo magnetic resonance imaging (MRI) since preoperative measurements of pancreatic fat by MRI offer noninvasive prediction of the occurrence of POPF. In an attempt to predict pancreatic texture preoperatively, Kuwahara $\mathrm{T}$ et al. [24] analysed the usefulness of EUS-EG in 59 patients, 26 with soft pancreas and 33 with hard pancreas, and revealed that the mean elasticity of the pancreas measured by EUS-EG $(>70)$ was an independent predictor of POPF. Although MRI and EUS-EG are considered useful tools, the usage of these modalities is still limited for the preoperative assessment of PD; therefore, our predictors measurable by plain $\mathrm{CT}$ are considered feasible and reasonable for predicting POPF.

Taken together, the high pancreas-visceral fat CT value ratio, serrated-type pancreas and these combinations could be associated with the infiltration of adipose tissue into the pancreas, resulting in parenchymal frailty. The frailty of the parenchyma makes it difficult to perform pancreaticojejunostomy and induces the vulnerability of anastomosis regardless of the surgeon's anastomosis technique. Therefore, we established a new strategic schema (Fig. 6) for the precise prediction of POPF preoperatively, which might contribute to the proactive approach for POPF.

\section{Conclusions}

The pancreas-visceral fat $\mathrm{CT}$ value ratio $>=-0.40$ and serrated-type pancreas allowed us to preoperatively identify a true POPF high-risk group regardless of pancreatic texture. Preoperative identification of a POPF high-risk group enabled us to develop a proactive strategy, such as the administration of somatostatin analogues and early follow-up CT scans for preventing POPF or its aggravation and for early detection. To establish the new strategy for preventing POPF for high-risk patients is imperative to improve a surgical outcome of PD.

\section{Abbreviations}

POPF: Postoperative pancreatic fistula; PD: Pancreatoduodenectomy; PWST: Pair-watch suturing technique; MPD): Main pancreatic duct; CT: Computed tomography; EUS-EG: Endoscopic ultrasonographyelastography; DM: Diabetes mellitus; PPPD: Pylorus-preserving PD; POD: Postoperative day; BL: Biochemical leak; ROls: Regions of interest; BMI: Body mass index; PV: Portal vain; ROC: Receiver-operating characteristic; IPMN: Intraductal papillary mucinous neoplasm; HU: Hounsfield Unit; PDAC: Pancreatic ductal adenocarcinoma; PI area: Parenchymal and interlobular; IMAC: Intramuscular adipose tissue content; MRI: Magnetic resonance imaging

\section{Acknowledgements}

Not applicable.

\section{Authors' contributions}

TK analyzed and drafted the manuscript. HK and SI participated data collection and assisted with data interpretation. YI, DN, KG, AH, TF, YM, AT, NK, YA, MK, SM, MU and HS reviewed and revised the manuscript. All authors read and approved the final manuscript.

\section{Funding}

Not applicable.

\section{Availability of data and materials}

The datasets generated and/or analyzed during the current study are not publicly available due to the data is confidential patient data but are available from the corresponding author on reasonable request.

\section{Ethics approval and consent to participate}

This retrospective study was approved by the ethics committee of Mie University Hospital and was conducted in accordance with the principles of the Declaration of Helsinki. As a retrospective cohort study, ethics committee waived the need for informed consent and there was an opt-out method.

\section{Consent for publication}

Not applicable.

\section{Competing interests}

The authors declare that they have no competing interests.

Received: 25 November 2019 Accepted: 31 May 2020

Published online: 11 June 2020

\section{References}

1. Cameron JL, Riall TS, Coleman J, Belcher KA. One thousand consecutive pancreaticoduodenectomies. Ann Surg. 2006;244:10-5.

2. De Oliveira ML, Winter JM, Schafer M, Cunningham SC, Cameron JL, Yeo CJ, et al. Assessment of complications after pancreatic surgery: a novel grading system applied to 633 patients undergoing pancreaticoduodenectomy. Ann Surg. 2006;244:931-9 discussion 937-9. 
3. Kimura W, Miyata H, Gotoh M, Hirai I, Kenjo A, Kitagawa Y, et al. A pancreaticoduodenectomy risk model derived from 8575 cases from a national single-race population (Japanese) using web-based data entry system: the 30-day and in-hospital mortality rates for pancreaticoduodenectomy. Ann Surg. 2014;259:773-80.

4. Kawai M, Kondo S, Yamaue H, Wada K, Sano K, Motoi F, et al. Predictive risk factors for clinically-relevant pancreatic fistula analyzed in 1,239 patients with pancreaticoduodenectomy: multicenter data collection as a project study of pancreatic surgery by the Japanese Society of Hepato- BiliaryPancreatic Surgery. J Hepatobiliary Pancreat Sci. 2011;18:601-8.

5. Ansorge C, Strömmer L, Andrén-Sandberg Å, Lundell L, Herrington MK, Segersvärd R. Structured intraoperative assessment of pancreatic gland characteristics in predicting complications after pancreaticoduodenectomy. Br J Surg. 2012;99:1076-82.

6. El Nakeeb A, Salah T, Sultan A, El Hemaly M, Askr W, Ezzat H, et al. Pancreatic anastomotic leakage after pancreaticoduodenectomy. Risk factors, clinical predictors, and management (single center experience). World J Surg. 2013;37:1405-18.

7. Grobmyer SR, Kooby D, Blumgart LH, Hochwald SN. Novel pancreaticojejunostomy with a low rate of anastomotic failure-related complications. J Am Coll Surg. 2010;210:54-9.

8. Fujii T, Sugimoto H, Yamada S, Kanda M, Suenaga M, Takami H, et al. Modified Blumgart anastomosis for pancreaticojejunostomy: technical improvement in matched historical control study. J Gastrointest Surg. 2014; 18:1108-15.

9. Hashimoto $Y$, Traverso LW. Incidence of pancreatic anastomotic failure and delayed gastric emptying after pancreatoduodenectomy in 507 consecutive patients: use of a web-based calculator to improve homogeneity of definition. Surgery. 2010;147:503-15.

10. Strasberg SM, Drebin JA, Soper NJ. Evolution and current status of the Whipple procedure: an update for gastroenterologists. Gastroenterology. 1997;113:983-94.

11. Yang YM, Tian XD, Zhuang Y, Wang WM, Wan YL, Huang YT. Risk factors of pancreatic leakage after pancreaticoduodenectomy. World J Gastroenterol. 2005;28(11):2456-61.

12. Kollmar O, Moussavian MR, Bolli M, Richter S, Schilling MK. Pancreatojejunal leakage after pancreas head resection: anatomic and surgeon-related factors. J Gastrointest Surg. 2007;11:1699-703.

13. Winter JM, Cameron JL, Campbell KA, Chang DC, Riall TS, Schulick RD, et al. Does pancreatic duct stenting decrease the rate of pancreatic fistula following pancreaticoduodenectomy? Results of a prospective randomized trial. J Gastrointest Surg. 2006;10:1280-90.

14. Bassi C, Falconi M, Molinari E, Mantovani W, Butturini G, Gumbs AA, et al. Duct-to-mucosa versus end-to-side pancreaticojejunostomy reconstruction after pancreaticoduodenectomy: results of a prospective randomized trial. Surgery. 2003;134:766-71.

15. Yeo CJ, Cameron JL, Maher MM, Sauter PK, Zahurak ML, Talamini MA, et al. A prospective randomized trial of pancreaticogastrostomy versus pancreaticojejunostomy after pancreaticoduodenectomy. Ann Surg. 1995; 222:580-8 discussion 588-592.

16. Lin JW, Cameron JL, Yeo CJ, Riall TS, Lillemoe KD. Risk factors and outcomes in postpancreaticoduodenectomy pancreaticocutaneous fistula. J Gastrointest Surg. 2004;8:951-9.

17. Cullen JJ, Sarr MG, Ilstrup DM. Pancreatic anastomotic leak after pancreaticoduodenectomy: incidence, significance, and management. Am J Surg. 1994;168:295-8.

18. Azumi Y, Isaji S, Kato H, Nobuoka Y, Kuriyama N, Kishiwada M, et al. A standardized technique for safe pancreaticojejunostomy: pair-watch suturing technique. World J Gastrointest Surg. 2010;27:260-4.

19. Azumi Y, Isaji S. Stented pancreaticojejunostomy (with video). J Hepatobiliary Pancreat Sci. 2012;19:116-24.

20. lizawa Y, Kato H, Kishiwada M, Hayasaki A, Tanemura A, Murata Y, et al. Long-term outcomes after pancreaticoduodenectomy using pair-watch suturing technique: different roles of pancreatic duct dilatation and remnant pancreatic volume for the development of pancreatic endocrine and exocrine dysfunction. Pancreatology. 2017;17:814-21.

21. Sugimoto M, Takahashi S, Kojima M, Kobayashi T, Gotohda N, Konishi M. In patients with a soft pancreas, a thick parenchyma, a small duct, and fatty infiltration are significant risks for pancreatic fistula after pancreaticoduodenectomy. J Gastrointest Surg. 2017;21:846-54.
22. Sugimoto M, Takahashi S, Gotohda N, Kato Y, Kinoshita T, Shibasaki H, et al. Schematic pancreatic configuration: a risk assessment for postoperative pancreatic fistula after pancreaticoduodenectomy. J Gastrointest Surg. 2013; 17:1744-51.

23. Yokoyama Y, Ebata T, Igami T, Sugawara G, Ando M, Nagino M. Proposal for a pancreatic configuration index for determining patients at high risk of pancreatic fistula following pancreatoduodenectomy. Dig Surg. 2016;33: 169-76.

24. Kuwahara T, Hirooka Y, Kawashima H, Ohno E, Yokoyama Y, Fujii T, et al. Usefulness of endoscopic ultrasonography-elastography as a predictive tool for the occurrence of pancreatic fistula afterpancreatoduodenectomy. J Hepatobiliary Pancreat Sci. 2017;24:649-56.

25. Al-Mrabeh A, Hollingsworth KG, Steven S, Taylor R. Morphology of the pancreas in type 2 diabetes: effect of weight loss with or without normalization of insulin secretory capacity. Diabetologia. 2016;59:1753-9.

26. Bassi C, Marchegiani G, Dervenis C, Sarr M, Abu Hilal M. Adham M, et al; the 2016 update of the international study group (ISGPS) definition and grading of postoperative pancreatic fistula: 11 years after. Surgery. 2017;161:584-91.

27. Rasband, W.S., ImageJ, U. S. National Institutes of Health, Bethesda, Maryland, USA, http://imagej.nih.gov/ij/, 1997-2012.

28. Callery MP, Pratt WB, Kent TS, Chaikof EL, Vollmer CM Jr. A prospectively validated clinical risk score accurately predicts pancreatic fistula after pancreatoduodenectomy. J Am Coll Surg. 2013;216:1-14.

29. Kitajima Y, Hyogo H, Sumida Y, Eguchi Y, Ono N, Kuwashiro T, et al. Japan nonalcoholic fatty liver disease study group (JSG-NAFLD). Severity of nonalcoholic steatohepatitis is associated with substitution of adipose tissue in skeletal muscle. J Gastroenterol Hepatol. 2013;28:1507-14.

30. Okumura S, Kaido T, Hamaguchi Y, Fujimoto Y, Masui T, Mizumoto M, et al. Impact of preoperative quality as well as quantity of skeletal muscle on survival after resection of pancreatic cancer. Surgery. 2015;157:1088-98.

31. Hamaguchi Y, Kaido T, Okumura S, Kobayashi A, Fujimoto Y, Ogawa K, et al. Muscle steatosis is an independent predictor of postoperative complications in patients with hepatocellular carcinoma. World J Surg. 2016;40:1959-68.

32. Takahashi H, Ogawa H, Ohigashi H, Gotoh K, Yamada T, Ohue M, et al. Preoperative chemoradiation reduces the risk of pancreatic fistula after distal pancreatectomy for pancreatic adenocarcinoma. Surgery. 2011;150: 547-56.

33. Denbo JW, Bruno ML, Cloyd JM, Prakash L, Lee JE, Kim M, et al. Preoperative Chemoradiation for pancreatic adenocarcinoma does not increase 90-day postoperative morbidity or mortality. J Gastrointest Surg. 2016;20:1975-85.

34. Pantic I, Nesic D, Stevanovic D, Starcevic V, Pantic S, Trajkovic V. Effects of ghrelin on the structural complexity of exocrine pancreas tissue architecture. Microsc Microanal. 2013;19:553-8.

35. Sasaki K, Asaoka T, Eguchi H, Fukuda Y, Iwagami Y, Yamada D, et al. Plasma ghrelin suppression as an early predictor for postoperative complications after pancreatoduodenectomy. Pancreatology. 2018;18:73-8.

36. Mathur A, Pitt HA, Marine M, Saxena R, Schmidt CM, Howard TJ, et al. Fatty pancreas: a factor in postoperative pancreatic fistula. Ann Surg. 2007;246: 1058-64.

37. Gaujoux S, Cortes A, Couvelard A, Noullet S, Clavel L, Rebours V, et al. Fatty pancreas and increased body mass index are risk factors of pancreatic fistula after pancreaticoduodenectomy. Surgery. 2010;148:15-23.

38. Lee SE, Jang JY, Lim CS, Kang MJ, Kim SH, Kim MA, et al. Measurement of pancreatic fat by magnetic resonance imaging: predicting the occurrence of pancreatic fistula after pancreatoduodenectomy. Ann.Surg. 2010;251:932-6.

\section{Publisher's Note}

Springer Nature remains neutral with regard to jurisdictional claims in published maps and institutional affiliations. 